\title{
EVALUACIÓN DE LOS COMPUESTOS FENÓLICOS Y CAPACIDAD ANTIOXIDANTE DE TRES COLORES DE PÉTALOS DE MASTUERZO (Tropaeolum majus L.)
}

\author{
Ketty Arellano Lino ${ }^{\text {a }}$ Jaqueline Herrera Rodríguez ${ }^{\mathrm{a}}$, Miguel Quispe Solano*a, Clara \\ Espinoza Silva ${ }^{\mathrm{b}}$, Nora Veliz Sedano ${ }^{\mathrm{b}}$, Wagner Orihuela Vásquez ${ }^{\mathrm{b}}$
}

\begin{abstract}
RESUMEN
Se evaluó el contenido de compuestos fenólicos y capacidad antioxidante en tres colores de pétalos de mastuerzo (Tropaeolum majus L.). Los pétalos de los colores de mastuerzo rojo, anaranjado y amarillo se secaron a temperatura ambiente; luego se acondicionaron para la extracción de sus componentes bioactivos, los que se cuantificaron mostrando diferencia significativa $(\mathrm{p}<0,05)$ entre colores. La mayor concentración de fenoles totales se encuentra en el color anaranjado, seguido por el rojo y el amarillo (917,05; 799,35 y 442,02 mg AGE/g de muestra). Para la cuantificación de flavonoides totales el mayor contenido se encuentra en el color rojo, seguido por el anaranjado y el amarillo $(2,184 ; 1,185$ y $0,867 \mathrm{mg} \mathrm{QE} / \mathrm{g}$ de muestra). En cuanto a los taninos totales, el mayor contenido se encuentra en el color rojo seguido por el anaranjado y el amarillo (342,92; 333,77 y 291,53 mg ATE/g de muestra). Con respecto a la capacidad antioxidante por los métodos DPPH y ABTS, el mayor contenido se encuentra en el color anaranjado, seguido por el rojo y amarillo $(3928,226 ; 3286,163$ y $2500,887 \mu \mathrm{M} \mathrm{TE} / \mathrm{g}$ de muestra), (622,92; 608,15 y 587,01 $\mu \mathrm{M} \mathrm{TE} / \mathrm{g}$ de muestra).
\end{abstract}

Palabras clave: Tropaeolum majus L., fenoles, flavonoides, taninos, capacidad antioxidante.

\section{EVALUATION OF PHENOLIC COMPOUNDS AND ANTIOXIDANT CAPACITY OF THREE COLOR PETAL CRESS (Tropaeolum majus L.)}

\begin{abstract}
The content of phenolic compounds and antioxidant capacity in three colors of petals cress (Tropaeolum majus L.) was evaluated. Petals colors red cress, orange and yellow were dried at room temperature; then they were conditioned for extracting its bioactive components, which were quantified showing significant difference $(p<0.05)$ between colors. The highest concentration of total phenols is in the orange color, followed by red and yellow (917.05; 799.35 and $442.02 \mathrm{mg} \mathrm{AGE} / \mathrm{g}$ sample). For quantification of total flavonoids, the highest content is in the red, followed by orange and yellow $(2.184 ; 1.185$ and $0.867 \mathrm{mg} \mathrm{QE} / \mathrm{g}$ sample). As for the total tannins, the highest content is in the red followed by orange and yellow (342.92; 333.77 and $291.53 \mathrm{mg}$ ATE/g sample). With respect to the antioxidant capacity by DPPH and ABTS methods, the highest content is in the orange color, followed

a Escuela Académico Profesional de Ingeniería Agroindustrial, Facultad de Ciencias Aplicadas, Universidad Nacional del Centro del Perú, quispe_miguelangel@hotmail.com

b Facultad de Ingeniería en Industrias Alimentarias, Universidad Nacional del Centro del Perú.
\end{abstract}


by red and yellow (3928.226; 3286.163 and $2500.887 \mu \mathrm{M}$ TE/g sample), (622.92; 608.15 and $587.01 \mu \mathrm{M}$ TE/g sample).

Key words: Tropaeolum majus L., phenols, flavonoids, tannins, antioxidant capacity.

\section{INTRODUCCIÓN}

El mastuerzo (Tropaeolum majus L.) es también conocido como capuchina, chaguinha, chagas y flor de sangre; pertenece a la diversidad primaria de América del Sur, se encuentra exclusivamente en Colombia, Brasil y Perú.

Presenta un alto contenido de vitamina $\mathrm{C}$, isotiocianatos, antocianinas, compuestos fenólicos: flavonoides, taninos. Estos compuestos fenólicos poseen una estructura química especialmente adecuada para ejercer una acción antioxidante. Además, debido a su reactividad, se encuentran en la mayoría de los casos combinadas con un ácido orgánico, un azúcar o bien, con ellas mismas para formar un polímero; están asociados con características sensoriales (color, sabor y astringencia), características nutrimentales y otras propiedades. Las flores de coloración pálida contienen menos polifenoles (blanca y lila claro) siendo su capacidad antioxidante menor ${ }^{1}$.

Tras un estudio a las flores de dalia se las consideró como un alimento funcional porque aportan compuestos fenólicos, especialmente las de coloración oscura, ya que presentaron la más alta composición fenólica y capacidad antioxidante ${ }^{2}$.

Muchas de estas flores contienen en sus pétalos componentes saludables y a su vez nos proporcionan calorías ${ }^{3}$. Esta funcionalidad se debe sobre todo a su capacidad antioxidante ${ }^{4}$ por contener elementos que en rosas se relacionan con compuestos fenólicos ${ }^{5}$ y en capuchina con isotiocianatos. Aunque la información aún es escasa, estos fitoquímicos serían protectores no necesarios para sobrevivir pero sí favorables para reducir el riesgo de padecer enfermedades coronarias y cancerígenas ${ }^{6}$.

Entre los métodos más aplicados para la determinación de la capacidad antioxidante están el ABTS y DPPH ambos presentan buena estabilidad en ciertas condiciones, aunque también muestran diferencias. El DPPH es un radical libre que puede obtenerse directamente sin una preparación previa, mientras que el ABTS tiene que ser generado tras una reacción que puede ser química, enzimática, o también electroquímica ${ }^{7}$. La complejidad de la mezcla de compuestos en un extracto, muchas veces requiere de condiciones especiales para poder medir la capacidad antioxidante, por esta razón existen varios métodos para su determinación ${ }^{8}$. 


\section{Materiales y reactivos}

\section{PARTE EXPERIMENTAL}

Se trabajó con tres colores de pétalos de mastuerzo (rojo, anaranjado y amarillo), figura 1 provenientes del anexo de Chipocayo, provincia de Tarma, departamento de Junín.

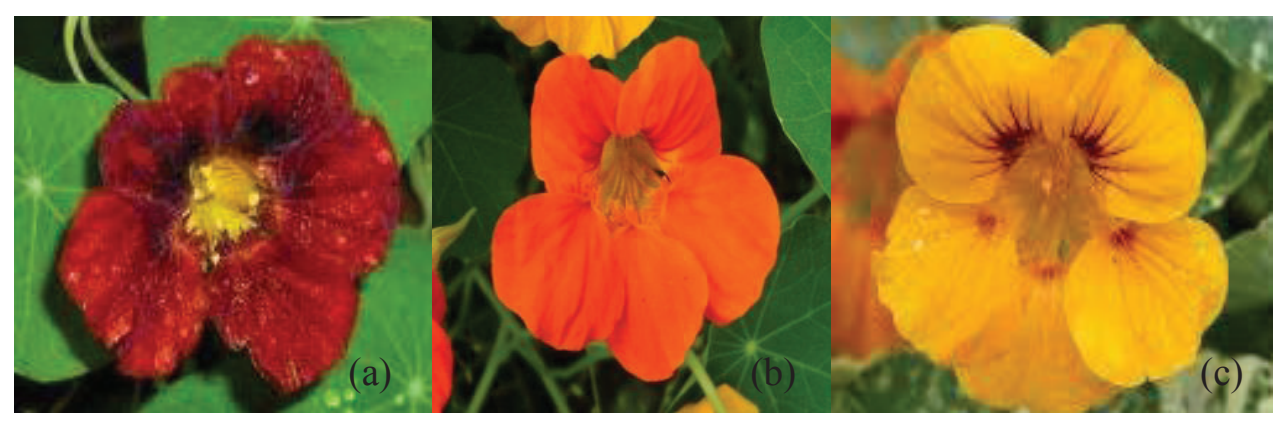

Figura 1. Flores de mastuerzo (Tropaeolum majus L.) (a) color rojo, (b) color anaranjado, (c) color amarillo.

\section{Obtención del extracto de pétalos del mastuerzo (Tropaeolum majus $L$.)}

El extracto es constituido exclusivamente de los pétalos de la flor para tal fin se prosiguió el diagrama de flujo siguiente (figura 2): 


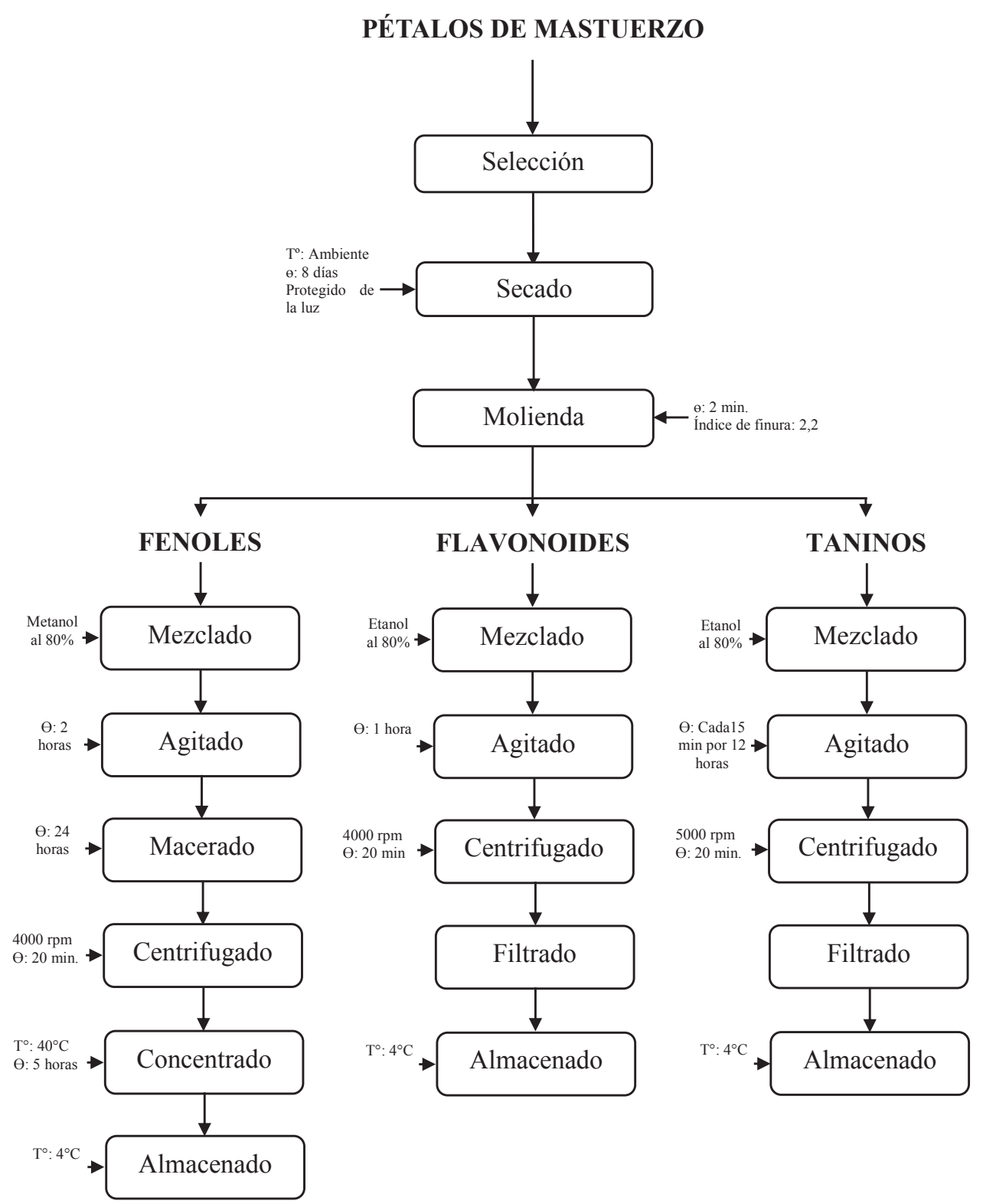

Figura 2. Diagrama de flujo de la obtención de los extractos de los pétalos de mastuerzo (Tropaeolum majus L.) para el análisis de fenoles, flavonoides y taninos totales. 


\section{Cuantificación de compuestos fenólicos y capacidad antioxidante}

Se cuantificó los compuestos fenólicos y capacidad antioxidante, para lo cual se realizó una curva estándar para cada análisis con reactivos y protocolos validados.

\section{Cuantificación de compuestos fenólicos}

Fenoles totales ${ }^{9}$

Flavonoides totales ${ }^{10}$

Taninos totales ${ }^{11}$

\section{Determinación de la capacidad antioxidante}

Método DPPH ${ }^{12}$

Método ABTS ${ }^{13}$

\section{Análisis estadístico.}

Para la evaluación estadística se utilizó un DCA cuyo análisis de varianza se trabajó con 0,05 de significancia; al encontrar diferencia significativa $(\mathrm{p}<0,05)$ se procedió a realizar la prueba de comparación de medias de Tukey. Los datos fueron procesados con la ayuda de los programas estadísticos Minitab y la hoja de cálculo Microsoft Excel 2010.

\section{RESULTADOS Y DISCUSIÓN}

Cuantificación de los compuestos fenólicos de los tres colores de pétalos del mastuerzo (Tropaeolum majus L.)

La cuantificación de los compuestos fenólicos de los colores de pétalos de mastuerzo (Tropaeolum majus L.) se efectuaron sobre extractos etanólicos y/o metanólicos de pétalos secos según los protocolos indicados.

\section{Cuantificación de fenoles totales}

Este método se basa en la reacción del extracto metanólico de los pétalos de mastuerzo con el Folin-Ciocalteu. Los resultados se reportan en la tabla 1.

Tabla 1. Contenido de fenoles totales, flavonoides y taninos en los tres colores de pétalos de mastuerzo (Tropaeolum majus L.).

\begin{tabular}{lccc}
\hline \multicolumn{1}{c}{ Componentes } & Color amarillo & Color anaranjado & Color rojo \\
\hline $\begin{array}{l}\text { Fenoles totales } \\
\text { (mg AGE/g de muestra) }\end{array}$ & $442,02 \pm 5,09^{\mathrm{b}}$ & $917,05 \pm 10,36^{\mathrm{a}}$ & $799,35 \pm 10,77^{\mathrm{a}}$ \\
$\begin{array}{l}\text { Flavonoides totales } \\
\text { (mg QE/g de muestra) }\end{array}$ & $0,867 \pm 0,022^{\mathrm{b}}$ & $1,185 \pm 0,025^{\mathrm{b}}$ & $2,184 \pm 0,022^{\mathrm{a}}$ \\
$\begin{array}{l}\text { Taninos totales } \\
\text { (mg ATE/g de muestra) }\end{array}$ & $291,53 \pm 5,63^{\mathrm{b}}$ & $333,77 \pm 9,75^{\mathrm{a}}$ & $342,92 \pm 24,26^{\mathrm{a}}$ \\
\hline
\end{tabular}

Valores con diferentes letras (a y b) dentro de cada columna denotan significancia en la prueba de Tukey $(\mathrm{p}<0,05)$, valores promedio de 3 repeticiones \pm desviación estándar. 
En la tabla 1 se reporta los resultados de las concentraciones de fenoles totales expresados en mg de ácido gálico equivalente/g de muestra; en la misma línea de investigación Gracia ${ }^{14}$ reporta un contenido de 221,481 $\pm 0,630 \mathrm{mg}$ de $\mathrm{AGE} / \mathrm{g}$ de muestra para el extracto de la flor de manita; Tarhan et al. ${ }^{15}$, en las flores de Curcubita pepo señalan valores de $189 \mathrm{mg}$ AGE/g de muestra cuyos valores se encuentran muy por debajo de los resultados obtenidos; esto se debe a la diferencia de colores de pétalos, pero que evidencian la presencia de compuestos fenólicos. En el caso de los flavonoides totales están expresados en mg de quercetina equivalente/g de muestra; Souto et al. ${ }^{16}$, encontró valores de 250,97; 134,76 y 123,83 mg QE/100 g de muestra para flores rojas, anaranjadas y amarillas de mastuerzo (Tropaeolum majus L.); estos resultados se encuentran cercanos a los obtenidos en la investigación. Finalmente, en el caso de los taninos totales están expresados en $\mathrm{mg}$ de ácido tánico equivalente/g de muestra; Márcia De Matos ${ }^{17}$ reportó el análisis de las flores de caléndula cuyos resultados en porcentaje son valores entre 10,4 y 11,2\% del total; a diferencia de éstos los taninos totales de los pétalos de mastuerzo presentaron mayor contenido con valores que figuran entre $29,15 \%$ a $34,29 \%$ para los tres colores evaluados.

\section{Determinación de la capacidad antioxidante de los tres colores de pétalos del mastuerzo (Tropaeolum majus L.)}

La caracterización de la capacidad antioxidante de los tres colores de pétalos de mastuerzo (Tropaeolum majus L.) se efectuó sobre extractos metanólicos de pétalos secos. Los resultados se han valorado con respecto al Trolox, utilizando dos metodologías: DPPH y ABTS, tal como se muestran en la tabla 2.

Tabla 2. Capacidad antioxidante por DPPH y ABTS de los extractos de pétalos de tres variedades de flor de mastuerzo (Tropaeolum majus L.).

\begin{tabular}{lccc}
\hline \multicolumn{1}{c}{ Componentes } & Color amarillo & Color anaranjado & Color rojo \\
\hline Método DPPH & $3286,163 \pm 9,00^{\mathrm{b}}$ & $3928,226 \pm 0,00^{\mathrm{a}}$ & $2500,887 \pm 49,30^{\mathrm{c}}$ \\
$(\mu \mathrm{M}$ TE/g de muestra) & & & \\
$\begin{array}{l}\text { Método ABTS } \\
(\mu \mathrm{M} \text { TE/g de muestra })\end{array}$ & $587,01 \pm 1,04^{\mathrm{b}}$ & $622,92 \pm 0,54^{\mathrm{a}}$ & $608,15 \pm 12,33^{\mathrm{a}}$ \\
\hline
\end{tabular}

Valores con diferentes letras ( $\mathrm{a}$, b y c) dentro de cada columna denotan significancia en la prueba de Tukey $(\mathrm{p}<0,05)$, valores promedio de 3 repeticiones \pm desviación estándar.

En la tabla 2 se reporta los resultados de la capacidad antioxidante de los tres colores de pétalos de mastuerzo (Tropaeolum majus L.) expresados en $\mu \mathrm{M}$ de Trolox equivalente/g de muestra, donde se puede apreciar que existe diferencia estadística $(\mathrm{p}<0,05)$.

Con respecto al método por DPPH, en trabajos similares se obtuvo resultados de $1507 \pm 86,2$ y $2651 \pm 93,5 \mu \mathrm{M}$ TE/g de muestra en el análisis a las hojas y tallos del guanandí (Calophyllum brasiliense) $)^{18}$, estos valores se encuentran por debajo de los obtenidos en la investigación 
con respecto al color rojo, anaranjado y amarillo. Para el caso del método por ABTS, en investigaciones similares se analizó las hojas del limoncillo donde se reportó un valor de $142,8 \mu \mathrm{M} \mathrm{TE} / \mathrm{g}$ extracto $^{19}$; este resultado es menor a los obtenidos en esta investigación.

Con el ABTS se puede medir los compuestos de naturaleza lipofílica como hidrofílica, mientras que el DPPH solo puede disolverse en medio orgánico, el primero es el más indicado para ensayos de compuestos coloreados, reduciendo posibilidades de interferencias de compuestos coloreados que absorben en la región del visible o compuestos resultantes de una reacción secundaria ${ }^{12}$. Los resultados obtenidos con DPPH y ABTS permiten alcanzar conclusiones prácticamente similares, ya que en ambos casos los pétalos de mastuerzo de color anaranjado obtuvieron resultados mayores, sin embargo al método ABTS es el más viable para el análisis de la capacidad antioxidante los diferentes extractos coloreados de los pétalos de mastuerzo (Tropaeolum majus L.).

\section{CONCLUSIONES}

- Se determinó que existe diferencia entre los colores de pétalos de mastuerzo (Tropaeolum majus L.), con respecto al contenido de compuestos fenólicos y capacidad antioxidante.

- Con respecto a la cuantificación de fenoles totales en los pétalos de mastuerzo la mayor concentración se encuentra en el color anaranjado con 917,05, seguido por el rojo con 799,35 y el amarillo con 442,02 mg AGE/g de muestra. En cuanto a la cuantificación de flavonoides totales el mayor contenido se encuentra en el color rojo con 2,184, seguido por el anaranjado con 1,185 y el amarillo con 0,867 mg QE/g de muestra. En lo que se refiere a la cuantificación de taninos totales, el mayor contenido se tiene en el color rojo con 342,92 , seguido por el anaranjado con 333,77 y finalmente, el amarillo con 291,53 $\mathrm{mg}$ ATE/g de muestra.

- En cuanto a la capacidad antioxidante por método de DPPH se obtuvo que la mayor capacidad antioxidante se encuentra en el color anaranjado con 3928,226, seguido por el amarillo con 3286,163 y el rojo con $2500,887 \mu \mathrm{M}$ TE/g de muestra; con respecto al método ABTS se reportó que el color anaranjado tiene 622,92, seguido por el rojo con 608,15 y finalmente, el amarillo con 587,01 $\mu \mathrm{M}$ TE/g de muestra.

- La valoración del contenido de fenoles totales, flavonoides, taninos y capacidad antioxidante en los tres colores de pétalos de mastuerzo (Tropaeolum majus L.) podría constituir una alternativa de uso en la industria farmacéutica y alimentaria.

\section{AGRADECIMIENTO}

Los autores expresamos nuestro agradecimiento a Dios. Al Instituto de Investigación de la UNCP, a la Facultad de Ciencias Aplicadas de la UNCP y a los Fondos del Canon Minero administrados por la Universidad Nacional del Centro del Perú.

\section{BIBLIOGRAFÍA}

1. K. Shindo, E. Saito, M. Sekiya, "Antioxidative activity of de flower of Torenia fournieri", 
Revista de Medicinas Naturales, 2007; 62(10): 247-258.

2. E. Lara, O. Martín, P. Osorio, L. Barrera, J. Sánchez, S. Bautista, “Actividad antioxidante composición nutrimental y funcional de flores comestibles de dalia", Revista Chapingo Serie Horticultura, 2014; 20(1): 101-116.

3. H. Friedman, "Tropaeolum majus L. as edible flowers growth and postharvest handling", Advances in Horticultural Science Journal's, 2005; 19(1): 3-8.

4. A. Vidal, A. Fallarero, E. Silva, A. Mara, A. De Lima, R. Pavan, et. al, "Composición química y actividad antioxidante del alga marina roja Bryothamnion triquetrum (S.G.Gmelin) Howe", Revista Brasileña de Ciencias Farmacéuticas, 2006; 42(4): 589600 .

5. T. Vanderjagt, R. Ghattas, D. Vanderjagt, M. Crossey, R. Glew, "Comparison of the total antioxidant content of 30 widely used medicinal plants of New México", Journal Life Sci, 2002; 70(9): 1035-1040.

6. N. Carazo, "Actividad antioxidante de los pétalos de flores comestibles", presentación oral, VI Congreso Ibérico de Ciencias Hortícolas y XII Congreso Nacional de Ciencias Hortícolas, Barcelona - España, 2005.

7. M. Arnao, "Some methodological problems in the determination of antioxidant activity using chromogen radicals: a practical case", Trends in Food Science \& Tecnology, 2000; 11(11): 419-421.

8. R. Prior, X. Wu, K. Schaich, "Standardized Methods for the Determination of Antioxidant Capacity and Phenolics in Foods and Dietary Supplements", Journal of the Agricultural and Food Chemistry, 2005; 53(10): 4290-4302.

9. L. Palomino, C. García, J. Gil, B. Rojano, D. Durango, "Determinación del contenido de fenoles y evaluación de la actividad antioxidante de propóleos recolectados en el departamento de Antioquia (Colombia)", Journal Vitae, 2009; 16(3): 388-395.

10. G. Pilco, "Comprobación del efecto adelgazante de la tintura de apio (Apium graveolens) y el perejil (Petroselinum sativum) en voluntarios con sobrepeso", tesis para optar el título de Químico Farmacéutico, Escuela Superior Politécnica de Chimborazo, 119, 2012.

11. Lastra, E. Rodríguez, H. Ponce de León, M. González, "Método analítico para la cuantificación de taninos en el extracto acuoso de romerillo", Revista Cubana de Plantas Medicinales, 2000; 5(1): 17-22.

12. E. Kuskoski, A. Asuero, A. Troncoso, J. Mancini-Filho, R. Fett, “Aplicación de diversos métodos químicos para determinar actividad antioxidante en pulpa de frutos", Revista Brasileña de Ciencia y Tecnología de Alimentos, 2005; 25(4):726-732.

13. M. Arnao, A. Cano, M. Acosta, "The hydrophilic and lipophilic contribution to total antioxidantactivity", Food Chemistry, 2001; 73(11): 239-244.

14. M. Gracia, http://www.uaq.mx/investigacion/difusion/veranos/memorias2007/56_1UAQGarciaNava.pdf (último acceso 17 de diciembre 2014).

15. L. Tarhan, H. Kayali, R. Urek, "In vitro antioxidant properties of Curcubita pepo L. male and female flowers extracts", Plant Foods for Human Nutrition, 2007; 62(2): 4951.

16. W. Souto, J. Alves, L. Cavalcante, "Capuchina (Tropaeolum majus L.)”, primera edición, Editorial Kiron, Brasilia, Brasil, 2012, 64. 
17. A. Márcia De Matos, "Avaliação do potencial antibacteriano de Calendula officinalis (Asteraceae) para seu emprego como fitoterápico", tesis para optar el título de Doctor en Ciencias, Universidad Federal de Paraná, 11, 2005.

18. A. Mesa, "Actividad antioxidante y contenido de fenoles totales de algunas especies del género Calophyllum", Revista Cubana de Plantas Medicinales, 2010; 15(2): 13-26.

19. B. Montoya, J. Lemeshko, A. López, A. Pareja, R. Torres, "Actividad antioxidante de algunos extractos vegetales", Journal Vitae, 2003; 10(2): 72-79. 